\title{
Energy-based predictions in Lorenz system by a unified formalism and neural network modelling
}

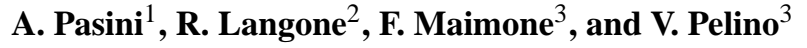 \\ ${ }^{1}$ CNR, Institute of Atmospheric Pollution Research, Rome, Italy \\ ${ }^{2}$ Katholieke Universiteit Leuven, Department ESAT/SISTA, Leuven, Belgium \\ ${ }^{3}$ Italian Air Force, CNMCA, Pratica di Mare (Rome), Italy
}

Received: 23 September 2010 - Accepted: 6 December 2010 - Published: 23 December 2010

\begin{abstract}
In the framework of a unified formalism for Kolmogorov-Lorenz systems, predictions of times of regime transitions in the classical Lorenz model can be successfully achieved by considering orbits characterised by energy or Casimir maxima. However, little uncertainties in the starting energy usually lead to high uncertainties in the return energy, so precluding the chance of accurate multi-step forecasts. In this paper, the problem of obtaining good forecasts of maximum return energy is faced by means of a neural network model. The results of its application show promising results.
\end{abstract}

\section{Introduction}

In the past few decades basic concepts of dynamical systems theory have been applied to a wide variety of real systems. One of the most important applications, for its impact on every-day life and society, has been in the field of numerical weather forecasts (Palmer and Hagedorn, 2006).

On the one hand the detailed study of several loworder chaotic systems has shed much light on their strange and unexpected behaviour, like the well-known fact that a small initial uncertainty can propagate in the course of time evolution even exponentially, making deterministic predictions about the final state possibly meaningless.

On the other hand the much more complex models like those used in operational meteorology gave no chance at all for a complete characterisation of their solutions, due to

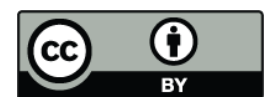

Correspondence to: A. Pasini

(pasini@iia.cnr.it) the impressively large number of variables and parameters involved. Such a characterisation would represent, in fact, a task well beyond the current state of computer technology.

Nevertheless, although questionable in principle, some of the concepts developed in the framework of low-order chaotic systems have been empirically transferred to the latter models, and have largely shown their usefulness for practical purposes.

Apart from operational meteorology, also climatology has benefited from elementary concepts of dynamical systems theory, such as that of "climate attractor" (see, e.g., Corti et al., 1999), whose existence for real climate system is a matter of philosophical controversy, and that of "climatic regimes" (see Stephenson et al., 2004, for a critical discussion on their definition and existence).

Motivated by new results obtained within the class of the so called Kolmogorov-Lorenz low-order models (Pasini and Pelino, 2000; Pelino and Pasini, 2001), which includes the famous Lorenz-63 model and a symmetric version of Lorenz84 model, in a recent paper (Pelino and Maimone, 2007) it has been proposed a possibly useful predictor, namely the intrinsic energy maximum, as a natural candidate to be used in forecasting various system's occurrences, such as regimes changes, mean lasting times in a given regime, and even, to some extent, the sequence of mean lasting times in the different regimes.

Multiple regimes and chaotic transition among them seem, in fact, to be a very common feature of dynamical systems, including fluid-dynamical ones, and the possible existence of approximate long-term predictors can be a desirable feature in many circumstances.

Published by Copernicus Publications on behalf of the European Geosciences Union and the American Geophysical Union. 
A first necessary step in view of a possible empirical application of the energetic argument to real systems, particularly within low-frequency variability of atmospheric patterns, in the same spirit of Kondrashov et al. (2004) where a Lorenz model is also used as a test case - and Deloncle et al. (2007), is to check whether a given statistical tool working solely on the solutions of the model (i.e., ignoring dynamical laws) is able to capture the relation between predictors and predictands in the system itself.

Aim of the present paper is to show that this is indeed possible using a neural network based technique.

\section{A unified formalism for Kolmogorov-Lorenz models and the role of maximum energy}

Let us start with a brief summary on Kolmogorov-Lorenz models. These models possess three degrees of freedom and their describing equations can be written as the sum of the Lie-Poisson brackets of the algebra of $\mathrm{SO}(3)$ spatial rotation group, and of dissipation and forcing terms (Einstein summation convention is used):

$\dot{x}_{i}=\left\{x_{i}, H\right\}-\Lambda_{i j} x_{j}+f_{i}, \quad(i=1,2,3)$

assuming the following gyrostat-like Hamiltonian

$H=\frac{1}{2} \Omega_{i k} x_{i} x_{k}+\boldsymbol{h}_{k} x_{k}$,

and the Lie-Poisson bracket structure

$$
\{F, G\}=J_{i k} \partial_{i} F \partial_{k} G, \text { with } J_{i k}=\varepsilon_{i j k} x_{j},
$$

where $\varepsilon_{i j k}$ is the Levi-Civita symbol representing the constants of structure of the algebra $\mathbf{g}=\operatorname{so}(3)$, and $F, G \in$ $C^{\infty}\left(\mathbf{g}^{*}\right)$ (Pasini and Pelino, 2000; Pelino and Pasini, 2001).

The main point on the above formalism is precisely the possibility to clearly separate dynamics into a Hamiltonian, a dissipation and a forcing contribution.

To be more specific, let us consider Lorenz-63 case, which is analysed in Pelino and Maimone (2007): the reader can refer to this paper for further details. In this particular case, Kolmogorov-Lorenz parameters become $\Omega=\operatorname{diag}(2,1,1)$ and $\boldsymbol{h}=(0,0,-\sigma)$ for the Hamiltonian part, $\Lambda=\operatorname{diag}(\sigma, 1, \beta)$ for the dissipation term and $\boldsymbol{f}=(0,0,-\beta(\rho+\sigma))$ for the forcing term. In the dynamical integrations we will perform here, the standard values $\sigma=10, \rho=28$ and $\beta=8 / 3$ will be considered.

The main peculiarity of the model's dynamics consists in the following: for a suitable choice of the parameters, a chaotic macro-dynamics can be identified, which consists of sudden and unpredictable transitions between two separate regions in phase-space, which will be referred to as the left $\left(\Psi_{\mathrm{L}}\right)$ and right $\left(\Psi_{\mathrm{R}}\right)$ regions covering the attractor $\Psi=$ $\Psi_{\mathrm{L}} \cup \Psi_{\mathrm{R}}$.

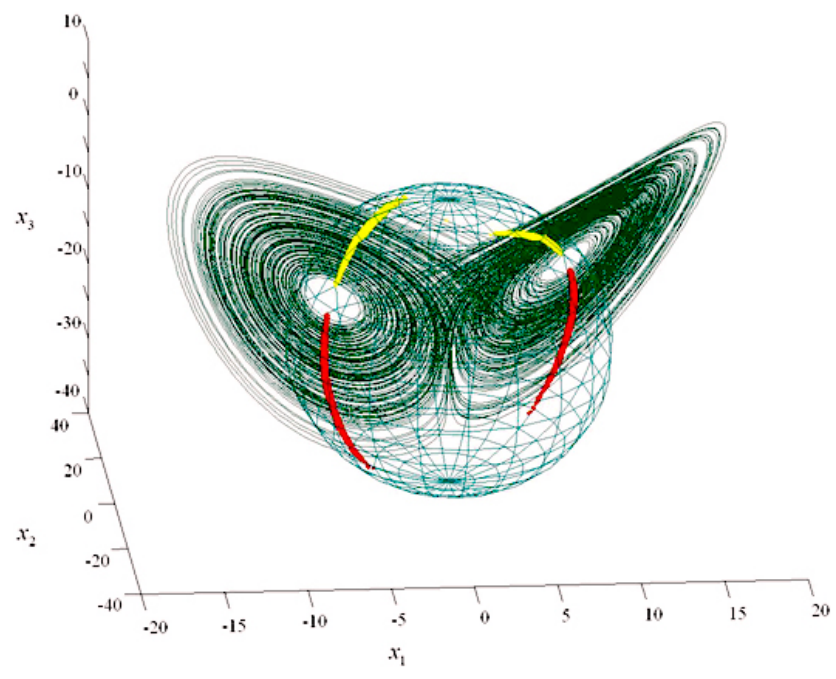

Fig. 1. Ellipsoid of Casimir extremes intersecting the attractor in four regions: the two stripes in the semi-space $x_{2}<0$ represent the set of maxima and the remaining two $\left(x_{2}>0\right)$ the minima. Figure from Pelino and Maimone (2007), copyright American Physical Society.

The intrinsic energy $E: \frac{1}{2} \Omega_{i k} x_{i} x_{k}+\boldsymbol{h}_{k} x_{k}=H$ defines a foliation of the phase-space into ellipsoid surfaces, while extreme values we are interested in are accomplished by the condition $\dot{H}=0$. By means of Eq. (1) one can see that this condition turns out in a geometric condition on the phase space, defining a new (fixed) ellipsoid.

This ellipsoid intersects $\Psi$ in four separate regions, which possess a natural fractal structure. Two of these regions correspond to minimum values of intrinsic energy and are located, by symmetry, each on a lobe of the attractor, while the other two ones represent the maximum values (as in Fig. 1).

A remarkable property of maximum energies, as opposed to the minimum ones, is that they have a natural order with respect to the distance from the fixed points in correspondence with each lobe. We can then consider the return map connecting points on a given region of maximum energies.

Another notable property of maximum energy regions is that they can be divided into ordered shells, each of which is associated with a given range of return time, and to a precise number of turns of the system's representative point on the opposite lobe. Moreover, it turns out that return times have a natural band structure, with the bands separated by a common distance of the order of $\tau_{0} \approx 0.66$ (for the given parameters).

On the other side, the knowledge of the starting shell of energy does not turn into a precise knowledge of the return shell, by which one could be allowed to re-iterate the forecast of the subsequent number of turns. However, shells themselves could be divided into sub-shells, each sub-shell 
corresponding to the number of turns that the representative point will perform after the second passage from the initial regime to the other one, and so on.

It is also worthwhile to note that what we have said for energy is also valid for Casimir function $C$, defined as the kernels of bracket (3), i.e. $\{C, G\}=0, \forall G \in C^{\infty}\left(\mathbf{g}^{*}\right)$. In the present case it is easy to check that $C=x_{k} x_{k}$. In Fig. 1 the maximum regions lying on the intersection between the maximum Casimir ellipsoid and the attractor are shown.

In summary, once known the energy of a starting point on a Casimir maximum at a certain lobe, the number of turns the system experiences in the other lobe is precisely determined. But, unfortunately, a little uncertainty in this starting energy is generally associated with a large uncertainty in the energy maximum on the first lobe when the trajectory is back: this fact does not allow us to perform a forecast for the next number of turns on the second lobe and achieve a multi-step forecast.

In the next section we will show that a learning algorithm is able to efficiently analyse the couples maximum energies - return times and to achieve just a reliable forecast of the energy maximum on the first lobe when the trajectory is back.

Dynamical integrations are performed via a 4th-order Runge-Kutta scheme with time step $\Delta t=0.01$, while for neural runs we use the model that will be sketched in the next section.

\section{A neural network model for empirical forecast of the return maximum energy}

During the last decades, neural networks (NNs) have shown their increasing usefulness in analysing many nonlinear systems, especially as far as the search for robust and reliable nonlinear relations between system variables (predictors and predictands) is concerned, even in presence of uncertainties and chaotic behaviours.

At present, many applications can be found in the realm of geophysical and environmental sciences: for recent reviews, see, for instance, Krasnopolsky (2007), Hsieh (2009) and some specific papers in Haupt et al. (2009). Furthermore, even several properties of the Lorenz-63 model have been analysed by means of NNs: see, for instance, de Oliveira et al. (2000), Boudjema and Cazelles (2001), Roebber and Tsonis (2005) and Pasini (2008). In this last recent paper, in particular, the difficulty of forecasting Lorenz variables in one-step by NN models is discussed, also through examples (see also references therein).

In this framework, the discovery of the particular role of maximum energy (inside the formalism sketched in the previous section) leads us to analyse if information about it and the return time allows a NN to establish a reliable relation with the return maximum energy. This could be the first step towards an extension of the prediction time-horizon on the Lorenz attractor.
Up to now, many kinds of NNs, endowed with distinct architectures and learning algorithms, have been developed. Here, for our investigation we adopt multi-layer perceptrons (MLPs) with a backpropagation training scheme.

General surveys of these networks can be found in Hertz et al. (1991), Bishop (1995) and Hsieh (2009), while the specific tool used in the present investigation has been worked out some years ago for diagnostic characterisation and forecast activity in complex systems (Pasini and Potestà, 1995; Pasini et al., 2001). Since its development, this tool has been applied to diagnostic and prognostic problems in the atmospheric boundary layer (Pasini and Potestà, 1995; Pasini et al., 2001, 2003; Pasini and Ameli, 2003), to attribution and impact analyses in the climate system (Pasini et al., 2006, 2009; Pasini and Langone, 2010) and to analyses of predictability in unforced and forced Lorenz models (Pasini, 2008).

The kernel of this tool consists in feed-forward networks with one hidden layer, characterised by nonlinear transfer functions (sigmoids) - calculated at hidden and output neurons - and a backpropagation training method with updating rules for weights including gradient descent and momentum terms. At each iteration step $t$, these rules read as follows:

$$
\begin{aligned}
W_{i j}(t+1) & =W_{i j}(t)-\eta \frac{\partial E^{\mu}}{\partial W_{i j}(t)}+m\left[W_{i j}(t)-W_{i j}(t-1)\right] \\
& =W_{i j}(t)+\eta \mathbf{g}_{i}^{\prime}\left(\boldsymbol{h}_{i}^{\mu}\right)\left(T_{i}^{\mu}-O_{i}^{\mu}\right) V_{j}^{\mu} \\
& +m\left[W_{i j}(t)-W_{i j}(t-1)\right]
\end{aligned}
$$

and

$$
\begin{aligned}
w_{j k}(t+1) & =w_{j k}(t)-\eta \frac{\partial E^{\mu}}{\partial w_{j k}(t)}+m\left[w_{j k}(t)-w_{j k}(t-1)\right] \\
& =w_{j k}(t)+\eta \mathbf{g}_{j}^{\prime}\left(\boldsymbol{h}_{j}^{\mu}\right) \sum_{i} W_{i j} \mathbf{g}_{i}^{\prime}\left(\boldsymbol{h}_{i}^{\mu}\right)\left(T_{i}^{\mu}-O_{i}^{\mu}\right) I_{k}^{\mu} \\
& +m\left[w_{j k}(t)-w_{j k}(t-1)\right]
\end{aligned}
$$

Here, referring to Eqs. (4)-(5) and Fig. 2, $W_{i j}$ and $w_{j k}$ are the weights associated to the "synapses" connecting hidden to output layer and input to hidden layer, respectively, $\mu$ is the number of a specific pattern of inputs-target couples, $I_{i}^{\mu}$ are the input data about predictors, $T_{i}^{\mu}$ are the targets, i.e. the real values of predictand data to be reconstructed/forecast by the $\mathrm{NN}, O_{i}^{\mu}$ are the outputs, i.e. the results of the NN in reconstructing/forecasting the targets, $\boldsymbol{h}_{j}^{\mu}$ and $\boldsymbol{h}_{i}^{\mu}$ are the weighted sums converging to the neurons of the hidden and output layers, respectively, $\mathbf{g}_{j}$ and $\mathbf{g}_{i}$ are the sigmoids calculated at hidden and output neurons, $V_{j}^{\mu}$ represent what exits from the hidden neurons after the calculation of their nonlinear transfer functions, $\mathbf{g}^{\prime}$ are the sigmoid derivatives, $\eta$ is the learning rate, directly associated with the minimization of the total error (a quadratic cost function of $T_{i}^{\mu}$ and $O_{i}^{\mu}$ ) on 


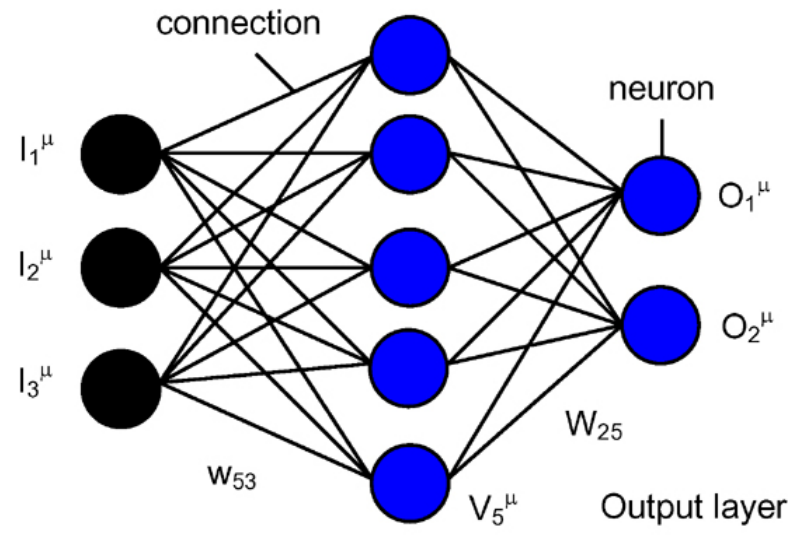

Input layer

Fig. 2. A feed-forward neural network.

the training set via gradient descent, and the term $m$ is the socalled momentum term, useful for avoiding large oscillations in the learning process. A good combination of $\eta$ and $m$ permits to escape from relative minima of the cost function and to reach a deeper minimum.

Once fixed the weights at the end of the iterative training (on a training set), the network is nothing but a function that maps input values to output ones. If this map shows its validity also for data which are unknown to the network (i.e. on validation/test sets), we have found a fully nonlinear regressive law linking input and output data (predictors and predictands). Of course, methods are to be used in order to prevent overfitting: here, early stopping is adopted.

Together with these quite common features of $\mathrm{NN}$ models, our tool provides also many training/validation/test procedures and facilities which are very useful for handling data from complex systems.

\section{NN runs and results}

By performing dynamical integrations of the Lorenz system, 66000 points characterised by maximum energies are obtained on the attractor. Then we calculate return time and maximum return energy for trajectories starting from these points. Thus every point can be labelled by a tripletpattern (maximum starting energy, return time, maximum return energy). We consider the first 44000 patterns as training set, the following 11000 as validation set and the last 11000 as test set for our NN runs.

As cited above, the main scope of using NNs in this framework is to analyse if information about maximum starting energies $\left(E_{\mathrm{st}}\right)$ on a lobe and return times permits to forecast the values of maximum return energy $\left(E_{\text {ret }}\right)$ on this lobe of the attractor when the trajectory is back. However, as a preliminary test of neural performance, it is interesting to analyse if NNs are able to predict the correct values of return time, starting from data about $E_{\text {st }}$.

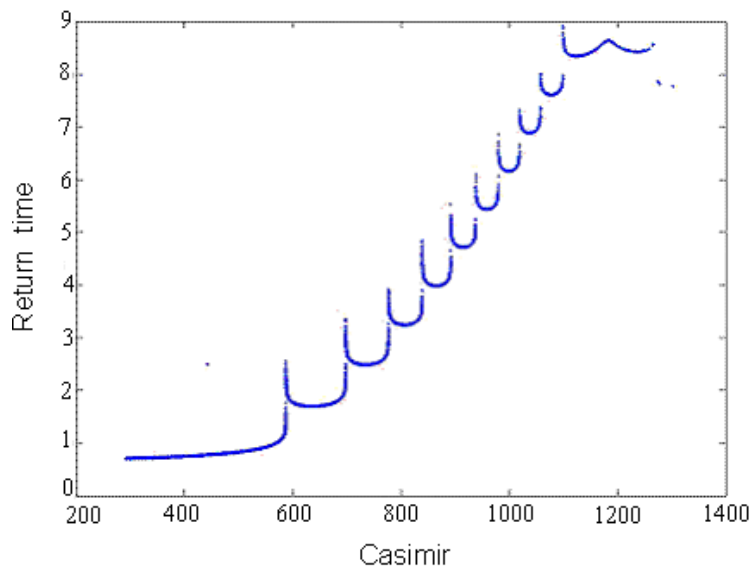

Fig. 3. Return times as a function of Casimir. Figure from Pelino and Maimone (2007), copyright American Physical Society.

Table 1. Performance of linear and NN predictions on the test sets summarised in terms of the linear correlation coefficient $R$ between targets and outputs.

\begin{tabular}{llll}
\hline $\begin{array}{l}\text { Input } \\
\text { variables }\end{array}$ & Target & $\begin{array}{l}\text { Linear } \\
\text { regression }\end{array}$ & $\begin{array}{l}\text { Neural } \\
\text { networks }\end{array}$ \\
\hline$E_{\text {st }}$ & Return time & 0.920 & $0.991 \pm 0.006$ \\
$E_{\text {st }}$ & $E_{\text {ret }}$ & 0.367 & $0.559 \pm 0.037$ \\
Return time & $E_{\text {ret }}$ & 0.109 & $0.702 \pm 0.026$ \\
$E_{\text {st }}+$ Return time & $E_{\text {ret }}$ & 0.679 & $0.960 \pm 0.019$ \\
\hline
\end{tabular}

From Pelino and Maimone (2007) it is known that these return times are quite precisely determined by the knowledge of $E_{\mathrm{st}}$ (or Casimir) and this is substantially due to a sort of "quantisation" of their values, as shown in Fig. 3: the "band structure" of the return times is well described in the same paper, too. As a matter of fact, a network endowed with $E_{\text {st }}$ as input, return time as target and 10 neurons in a single hidden layer is able to catch this relationships as shown, for instance, by the performance in predicting the values of return time on the test set, summarised in the first row of Table 1. In this table the error bars associated with $\mathrm{NN}$ forecasts are derived by ensemble runs of the model performed for networks with the same topology but endowed with different initial random weights, so that the networks themselves are able to widely explore the landscape of the cost function. The interval indicates \pm 2 standard deviations.

After this preliminary test, the main task of predicting the target values of maximum return energy is tackled. Some attempts are performed by considering just one predictor ( $E_{\mathrm{st}}$ or return time) at once. Even if the performance of these networks is much better than linear regressions (see Table 1), these results are not accurate enough and do not supply us with a satisfying forecast of the return energy 


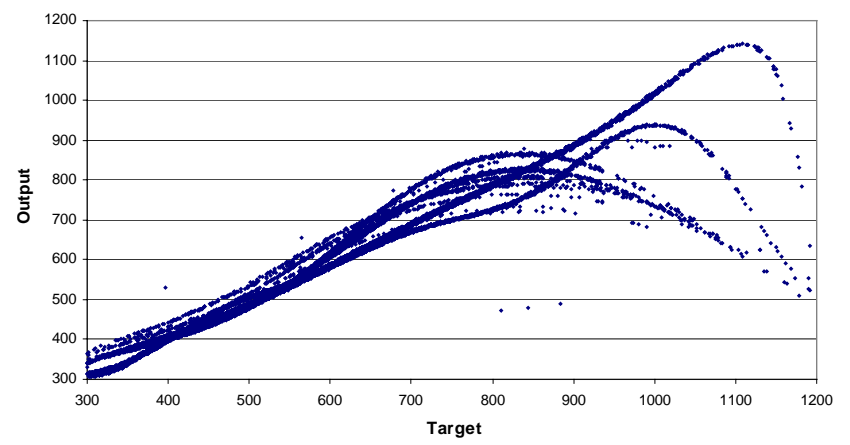

Fig. 4. Results of NN forecasts: outputs ( $E_{\text {ret }}$ predictions) vs. targets ( $E_{\text {ret }}$ real values) on the test set.

values. Thus, a new network characterised by topology 220-1 is now trained for this task. Here, $E_{\text {st }}$ and return time are the inputs (predictors) and $E_{\text {ret }}$ is the target (predictand). Empirical tests show that an amount of 20 neurons in a single hidden layer is enough to correctly capture the underlying function linking the data and not so high to lead us to fall into overfitting problems.

If Table 1 supplies us with an index of clear increase in performance, an analysis of Figs. 4-7 permits to achieve a deeper insight of the neural results.

In Fig. 4 the scatter plot of $\mathrm{NN}$ outputs ( $E_{\text {ret }}$ predictions) vs. targets ( $E_{\text {ret }}$ real values) is shown on the test set. Due to the high correlation coefficient shown in Table 1, one foresees a cloud of points quite closed to the bisecting line and somewhat homogeneous. As a matter of fact, the points are closed enough to the bisecting line, if we exclude the poorer performance for high energies, but, in any case, the graph seems quite strange: the cloud is not homogeneous and several preferred lines are visible. May we suppose this is due to the structure of our data, in particular to the "quantised" band structure of return times?

In order to test this hypothesis, the results of $E_{\text {ret }}$ predictions are divided into classes, one for each band of return times: in Fig. 5 the results for the first eight classes $(\# 1, \ldots, \# 8)$ are shown. It is now clear that each preferred line visible in Fig. 4 is associated with a different band of return times.

From Figs. 4-5 a decrease in performance for high energies, especially for high bands of return times, is also evident. The reason for this lack of performance can be twofold. First of all, in these regions we find just a few points and we have a poor statistics for the training of NNs. Secondly, the dynamics of the Lorenz system implies that at high starting energies a little change (error) in $E_{\text {st }}$ leads to a big change (error) in $E_{\text {ret }}$. Look at Fig. 6, where the blue squares show the dynamical law linking $E_{\text {st }}$ and $E_{\text {ret }}$ : the blue curves become more and more vertical as starting energy increases.

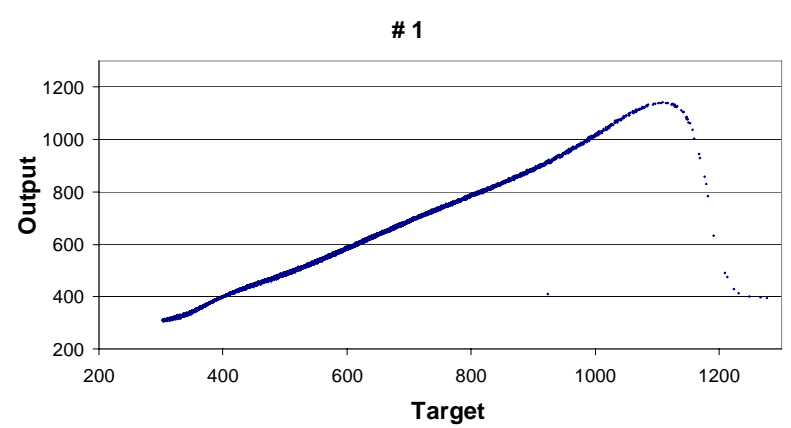

\# 2

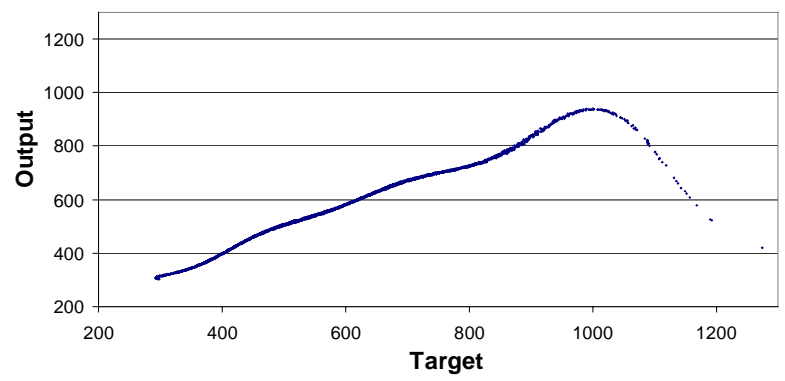

\# 3

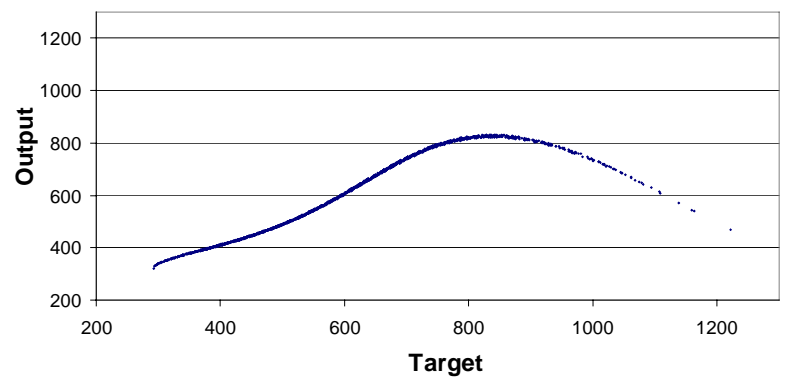

\# 4
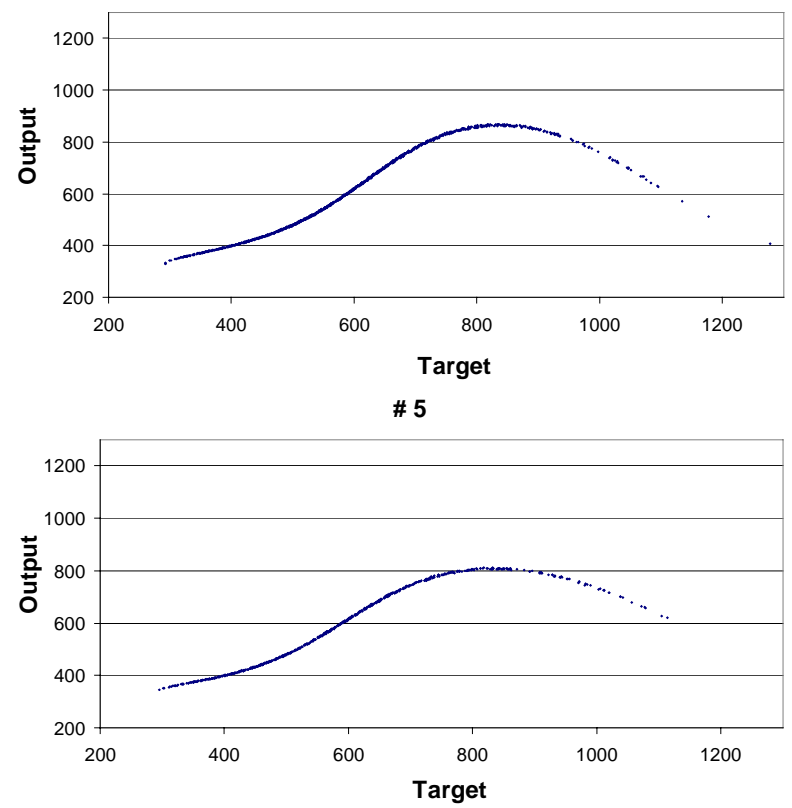

Fig. 5. Results of $\mathrm{NN}$ forecasts: outputs ( $E_{\text {ret }}$ predictions) vs. targets ( $E_{\text {ret }}$ real values) on the test set for the first eight classes of return-time bands. 

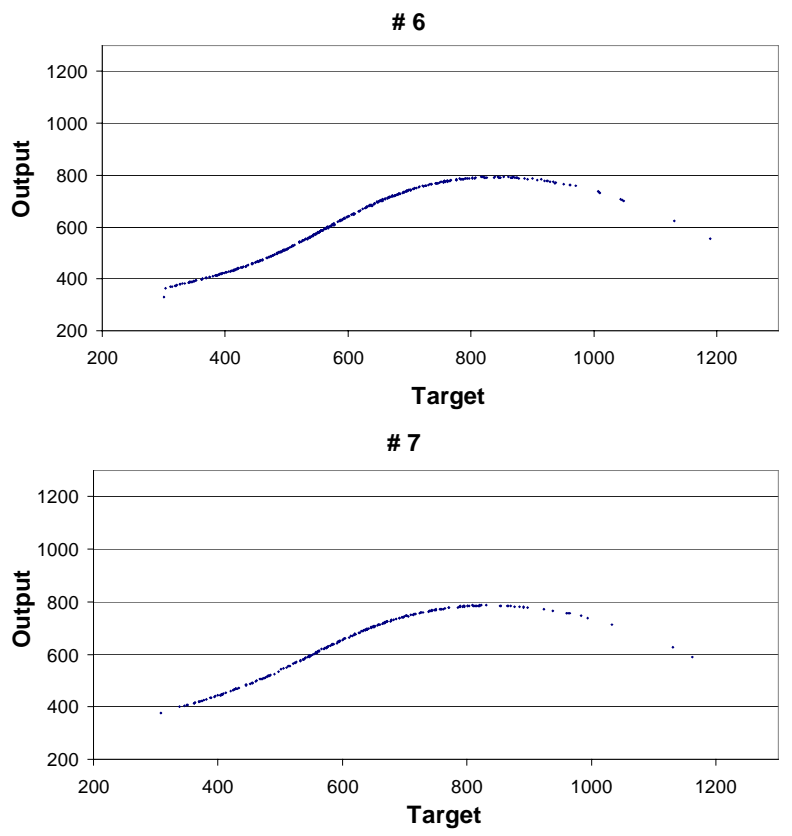

\# 8

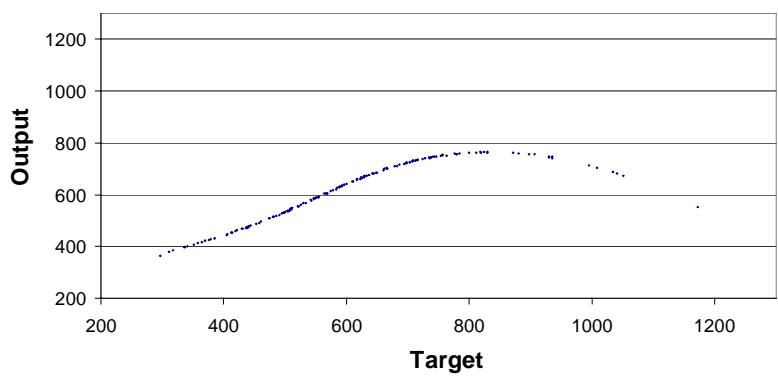

Fig. 5. Continued.

Also Fig. 6 helps us to recognise the goodness of NN forecasts and their correct reconstruction of the dynamical law for medium-low starting energies. Furthermore, this figure shows that the performance of $\mathrm{NN}$ nonlinear model overwhelms the poorest performance of the multi-linear regression.

Finally, in Fig. 7 the results of our 1-step forecast for return energy are presented on a specific subset. One can see that the prediction results cover almost completely the curve representing the dynamical relationship between $E_{\text {st }}$ and $E_{\text {ret }}$.

In summary, a simple NN model fed by data about maximum starting energy on a lobe and return time has shown its ability to predict the value of maximum return energy when the trajectory is back on the same lobe of the Lorenz system.

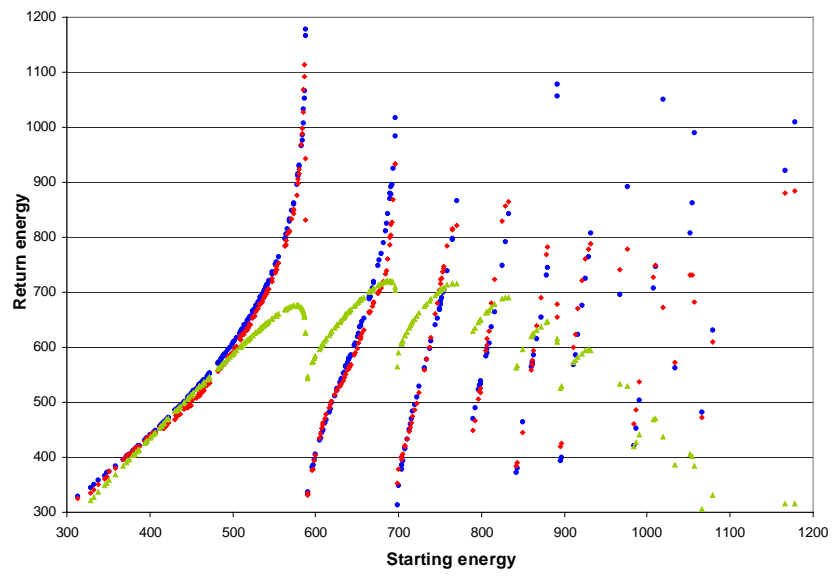

Fig. 6. Dynamical relation between starting energy maxima and return energy maxima (blue squares), $\mathrm{NN}$ forecasts (red diamonds) and multi-linear forecasts (green triangles) on a sample of the test set.

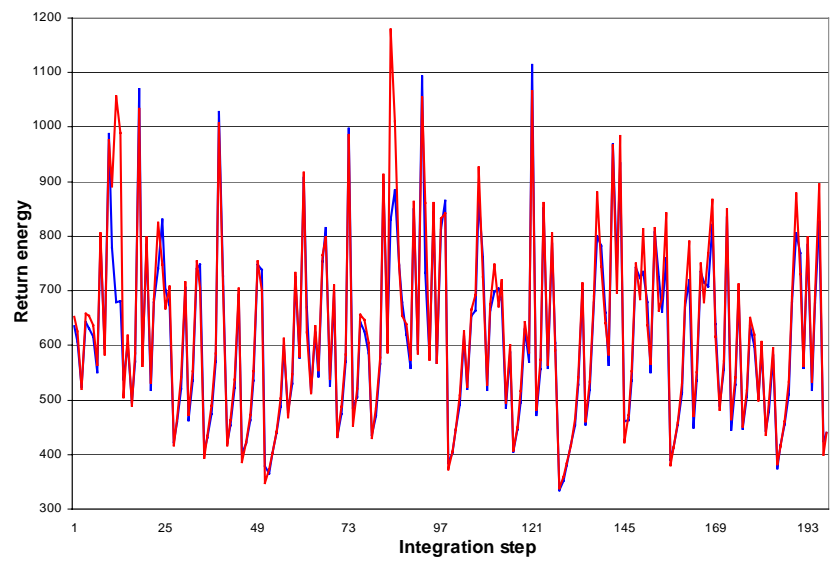

Fig. 7. Example of NN prediction (red line) of the real values of maximum return energy (blue line) on a subset of the test set.

\section{Conclusions and prospects}

In this paper, we adopted a unified formalism which previously showed the importance of considering energetic maxima for achieving an increased predictability in the Lorenz system. This formalism led to a quite precise determination of return times referred to the transitions from a lobe to the other one. Nevertheless, in Lorenz dynamics little uncertainties in starting energy are related very often to high uncertainties in return energy, so precluding successful iterated predictions of regime transitions.

In this chaotic framework, we analyse the chance of identifying an empirical tool which is able to capture the relation between maximum starting energy and maximum return energy, thus supplying us with a first correct step towards a possible long-term prediction of regime transitions 
on the Lorenz attractor. A NN model has shown good results in predicting return energy once fed by data about starting energy and return time.

Here, simple multi-layer perceptrons are applied and prediction is limited to 1-step forecasts. As prospects of further research, application of improved NN models is planned and multi-step forecasts are envisaged.

Furthermore, the formalism adopted here can be applied also in the case of more general and realistic N-dimensional fluid-dynamical systems, such as those discussed in Pelino and Maimone (2007). Thus, we are confident that our dynamical and NN approach can be pursued to catch some features of dynamics and predictability of the real atmosphere, too.

Acknowledgements. Two of us (F.M. and V.P.) were partially supported by PEPS Mathematical Methods of Climate Models.

Edited by: W. Hsieh

Reviewed by: two anonymous referees

\section{References}

Bishop, C. M.: Neural networks for pattern recognition, Oxford University Press, New York, USA, 1995.

Boudjema, G. and Cazelles, B.: Extraction of nonlinear dynamics from short and noisy time series, Chaos Soliton. Fract., 12, 20512069, doi:10.1016/S0960-0779(00)00163-6, 2001.

Corti, S., Molteni, F., and Palmer, T. N.: Signature of recent climate change in frequencies of natural atmospheric circulation, Nature, 398, 799-802, doi:10.1038/19745, 1999.

de Oliveira, K. A., Vannucci, A., and da Silva, E. C.: Using artificial neural networks to forecast chaotic time series, Physica A, 284, 393-404, doi:10.1016/S0378-4371(00)00215-6, 2000.

Deloncle, A., Berk, R., D’Andrea, F., and Ghil, M.: Weather regime prediction using statistical learning, J. Atmos. Sci., 64, 16191635, 2007.

Haupt, S. E., Pasini, A., and Marzban, C. (Eds.): Artificial intelligence methods in the environmental sciences, Springer, New York, USA, 2009.

Hertz, J., Krogh, A., and Palmer, R. G.: Introduction to the theory of neural computation, Addison-Wesley-Longman, Reading, MA, USA, 1999.

Hsieh, W. W.: Machine learning methods in the environmental sciences: Neural networks and kernels, Cambridge University Press, Cambridge, UK, 2009.

Kondrashov, D., Ide, K., and Ghil, M.: Weather regimes and preferred transition paths in a three-level quasi-geostrophic model, J. Atmos. Sci., 61, 568-587, 2004.
Krasnopolsky, V. M.: Neural network emulations for complex multidimensional geophysical mappings: Applications of neural network techniques to atmospheric and oceanic satellite retrievals and numerical modeling, Rev. Geophys., 45, RG3009, doi:10.1029/2006RG000200, 2007.

Palmer, T. and Hagedorn, R.: Predictability of Weather and Climate, Cambridge University Press, Cambridge, UK, 2006.

Pasini, A.: External forcings and predictability in Lorenz model: An analysis via neural network modelling, Nuovo Cimento C, 31, 357-370, 2008.

Pasini, A. and Ameli, F.: Radon short range forecasting through time series preprocessing and neural network modelling, Geophys. Res. Lett., 30(7), 1386, doi:10.1029/2002GL016726, 2003.

Pasini, A. and Langone, R.: Attribution of precipitation changes on a regional scale by neural network modeling: A case study, Water, 2, 321-332, doi:10.3390/w2030321, 2010.

Pasini, A. and Pelino, V.: A unified view of Kolmogorov and Lorenz systems, Phys. Lett. A, 275, 435-446, doi:10.1016/S03759601(00)00620-4, 2000.

Pasini, A. and Potestà, S.: Short-range visibility forecast by means of neural-network modelling: A case study, Nuovo Cimento C, 18, 505-516, doi:10.1007/BF02506781, 1995.

Pasini, A., Pelino, V., and Potestà, S.: A neural network modeling for visibility nowcasting from surface observations: Results and sensitivity to physical input variables, J. Geophys. Res., 106, 14951-14959, doi:10.1029/2001JD900134, 2001.

Pasini, A., Perrino, C., and Žujić, A.: Non-linear atmospheric stability indices by neural network modelling, Nuovo Cimento C, 26, 633-638, doi:10.1393/ncc/i2004-10001-7, 2003.

Pasini, A., Lorè, M., and Ameli, F.: Neural network modelling for the analysis of forcings/temperatures relationships at different scales in the climate system, Ecol. Model., 191, 58-67, doi:10.1016/j.ecolmodel.2005.08.012, 2006.

Pasini, A., Szpunar, G., Amori, G., Langone, R., and Cristaldi, M.: Assessing climatic influences on rodent density: A neural network modelling approach and a case study in Central Italy, Asia-Pac. J. Atmos. Sci., 45, 319-330, 2009.

Pelino, V. and Maimone, F.: Energetics, skeletal dynamics, and long-term predictions on Kolmogorov-Lorenz systems, Phys. Rev. E, 76, 046214, doi:10.1103/PhysRevE.76.046214, 2007.

Pelino, V. and Pasini, A.: Dissipation in Lie-Poisson systems and the Lorenz-84 model, Phys. Lett. A, 291, 389-396, doi:10.1016/S0375-9601(01)00764-2, 2001.

Roebber, P. J. and Tsonis, A. A.: A method to improve prediction of atmospheric flow transitions, J. Atmos. Sci, 62, 3818-3824, doi:10.1175/JAS3572.1, 2005.

Stephenson, D. B., Hannachi, A., and O'Neill, A.: On the existence of multiple climate regimes, Q. J. Roy. Meteor. Soc., 130, 583605, doi:10.1256/qj.02.146, 2004. 